\title{
Magnetic Resonance Imaging of Brain Death
}

\author{
Akira MATSUMURA*,†, Kotoo MEgURO*, Hideo TSURUSHIMA*, \\ Youji KoMATSU*, Yoichi KIKUCHI ${ }^{* *}$, Mitsuyoshi WADA**, \\ Yoshitaka NAKATA*, Noriyoshi OHASHI ${ }^{* * *}$, and Tadao NosE ${ }^{\dagger}$

\begin{abstract}
Departments of ${ }^{*}$ Neurosurgery, ${ }^{* *}$ Radiology, and ${ }^{* * *}$ Acute Medicine, Tsukuba Medical Center Hospital, Tsukuba, Ibaraki; ${ }^{\dagger}$ Department of Neurosurgery, Institute of Clinical Medicine, University of Tsukuba, Tsukuba, Ibaraki
\end{abstract}

\begin{abstract}
Fifteen patients with clinical diagnosis of brain death were examined by magnetic resonance (MR) imaging. Aortography with intraarterial digital subtraction angiography (IADSA) was also performed in nine patients. MR imaging indications of the flow void phenomenon were evaluated in the cavernous portion of internal carotid artery (ICA) and the middle or anterior cerebral artery, and compared with the IADSA findings. The relative intensities of gray and white matters were also measured. MR imaging showed that flow voids were absent in the ICA in all eight patients in whom non-filling was confirmed by IADSA. In one patient, IADSA demonstrated intracranial flow despite the diagnosis of brain death and the flow void pattern was normal. Serial MR imaging showed disappearance or abnormality of flow voids after brain death in six patients and absence before brain death in one. Spotty flow voids became visible in the unilateral ICA of one case after brain death. Partial residual flow voids may be caused by to-and-fro blood movement which was demonstrated by transcranial Doppler sonography. The normal flow void pattern was seen in none of these patients, therefore absence of flow voids indicates cessation of intracranial blood flow. Proton density and $T_{2}$-weighted MR images showed dissociated intensity changes between white and gray matters, which were thought to be characteristic of brain death. In conclusion, MR imaging can achieve non-invasive diagnosis of the non-filling phenomenon in patients with brain death.
\end{abstract}

Key words: brain death, magnetic resonance imaging, flow void, non-filling, angiography, diagnosis

\section{Introduction}

Brain death results in various morphological changes including massive brain swelling, and central and tonsillar herniation, which can be observed by computed tomography $(\mathrm{CT})^{13)}$ and magnetic resonance (MR) imaging. ${ }^{1}$ The diagnosis of brain death can be confirmed by demonstration of the absence of cerebral blood flow if other indicators are unclear or under evaluation. ${ }^{21)}$

Various radiological techniques can show the absence of cerebral blood flow as the non-filling phenomenon, including conventional angiography, ${ }^{15)}$ intraarterial digital subtraction angiography (IADSA), ${ }^{19,20)} \mathrm{CT}^{13)}$ dynamic $\mathrm{CT},{ }^{7,19}$ and radionuclide angiography. ${ }^{17)}$ Other techniques such as transcranial Doppler (TCD) have also been widely used. ${ }^{12.14)}$ MR imaging can demonstrate blood flow non-invasively without the use of contrast material ${ }^{4,8)}$ and shows intravascular blood flow in the main branches of the intracranial vessels as the flow void phenomenon. ${ }^{2,4,8)}$ Demonstration of the non-filling phenomenon by MR imaging, without using contrast medium or other invasive techniques, would facilitate the diagnosis of brain death.

Here we report a MR imaging and IADSA study to investigate the use of MR imaging in the diagnosis of brain death.

Received June 26, 1995; Accepted August 29, 1995

Author's present address: A. Matsumura, M.D., Department of Neurosurgery, Institute of Clinical Medicine, University of Tsukuba, Tsukuba, Ibaraki, Japan. 


\section{Materials and Methods}

\section{Patient profiles}

This study included eight male and seven female patients with clinical diagnosis of brain death aged from 20 to 78 years (mean $57 \mathrm{yrs}$ ). The primary diseases were subarachnoid hemorrhage (6 cases), severe head injury (4), intracerebral hemorrhage (3), and anoxic brain damage (2).

The diagnosis of brain death was based on the guidelines of the Ministry of Health and Welfare of Japan, which require that the diagnosis be based on a combination of neurological findings, electroencephalography, and apnea tests repeated at a minimum interval of 6 hours. In our patients, these tests were performed at an interval of 24 hours. The diagnosis of brain death was confirmed at the second evaluation.

\section{Flow void and non-filling detection}

MR imaging used a 0.5 Tesla superconducting MR system (MRT50A; Toshiba, Tokyo) using the spin echo (SE) sequence with a repetition time (TR) of $2000 \mathrm{msec}$ and echo time (TE) $30 \mathrm{msec}$ for proton density (PD) imaging and $80 \mathrm{msec}$ for $\mathrm{T}_{2}$-weighted imaging. The gradient field echo sequence was used for $T_{1}$-weighted imaging. These images were acquired consecutively (no interleaved scan) with a slice thickness of $10 \mathrm{~mm}$, without slice gap or flow compensation pulse.

All patients were examined under cardiorespiratory support and monitoring as described elsewhere. ${ }^{9}$ MR images were taken before and after the diagnosis of brain death in eight patients and only after the diagnosis of brain death in seven patients, depending on their clinical condition.

The presence or absence of flow voids was evaluated where the cavernous portion of the internal carotid artery (ICA) and the middle (MCA) or anterior cerebral artery (ACA) were visualized. The presence or absence of flow voids was compared with the IADSA findings.

IADSA was performed in nine patients to confirm the non-filling phenomenon in both carotid and vertebral arteries. A total dose of $20 \mathrm{ml}$ ioxaglic acid (Hexabrix; Tanabe Pharmaceutical, Osaka) was injected at $10 \mathrm{ml} / \mathrm{sec}$ using an infusator through a $4 \mathrm{~F}$ pig tail catheter. The angiograms were taken for 20 seconds. IADSA was performed on the same day as brain death diagnosis in three patients, 1 day later in two patients, more than 2 days later in four patients. No patient recovered after the diagnosis of brain death.

\section{Intraparenchymal signal intensity measure- ment}

The MR images were recorded as computer data in 10 patients (12 scans). The signal intensities of the white and gray matters were measured from the $\mathrm{SE}$ images (TR/TE $2000 / 30 \mathrm{msec}$ ) in a region of interest of $15 \mathrm{~mm} \times 15 \mathrm{~mm}$ at the level of the frontal white matter and at the basal ganglia bilaterally in the same slice. Eleven patients without intracranial lesions were also evaluated as controls. The data from patient and control groups were compared with Student's t-test.

\section{Results}

\section{Flow void and non-filling phenomena}

MR imaging showed no normal flow void phenomenon in any patient except one after the diagnosis of brain death. In the exceptional case, the intracranial blood flow was confirmed by IADSA. Ten of the 14 patients with abnormal flow void pattern showed complete disappearance of flow void in the cavernous ICA as well as MCA or ACA (Fig. 1). Three patients had flow void patterns as a spotty area in the branches of MCA or ACA, mostly in the perpendicular portion to the axial slices (Fig. 2), but the flow voids in the cavernous portion were absent. Unilateral spotty flow void pattern was seen in the ICA of one patient (no IADSA performed).

Combined MR imaging and IADSA confirmed the absence of both flow voids and blood flow at the cavernous ICA in eight patients. Among these eight patients, spotty flow voids were seen in the ACA or MCA in two patients.

Serial MR images before and after the diagnosis of brain death showed disappearance of flow voids at the cavernous portion after the diagnosis of brain death in six patients, and absence before and after the diagnosis in one patient. In one patient, flow void in the unilateral ICA appeared after the diagnosis of brain death. However, this occurred only as a small spot, not along the entire ICA as normally seen in preserved cerebral perfusion. IADSA could not be performed in this patient.

\section{Intraparenchymal signal intensity changes}

The ratio of signal intensities between white and gray matters increased after the diagnosis of brain death on PD and $\mathrm{T}_{2}$-weighted images (Fig. 3), but decreased on $T_{1}$-weighted images. On the $P D$ image (TR/TE $2000 / 30 \mathrm{msec}$ ), the intensity ratio was 1.23 \pm 0.098 in patients with brain death and $1.07 \pm$ 0.035 in control subjects. This difference was statistically significant $(\mathrm{p}<0.05)$. 

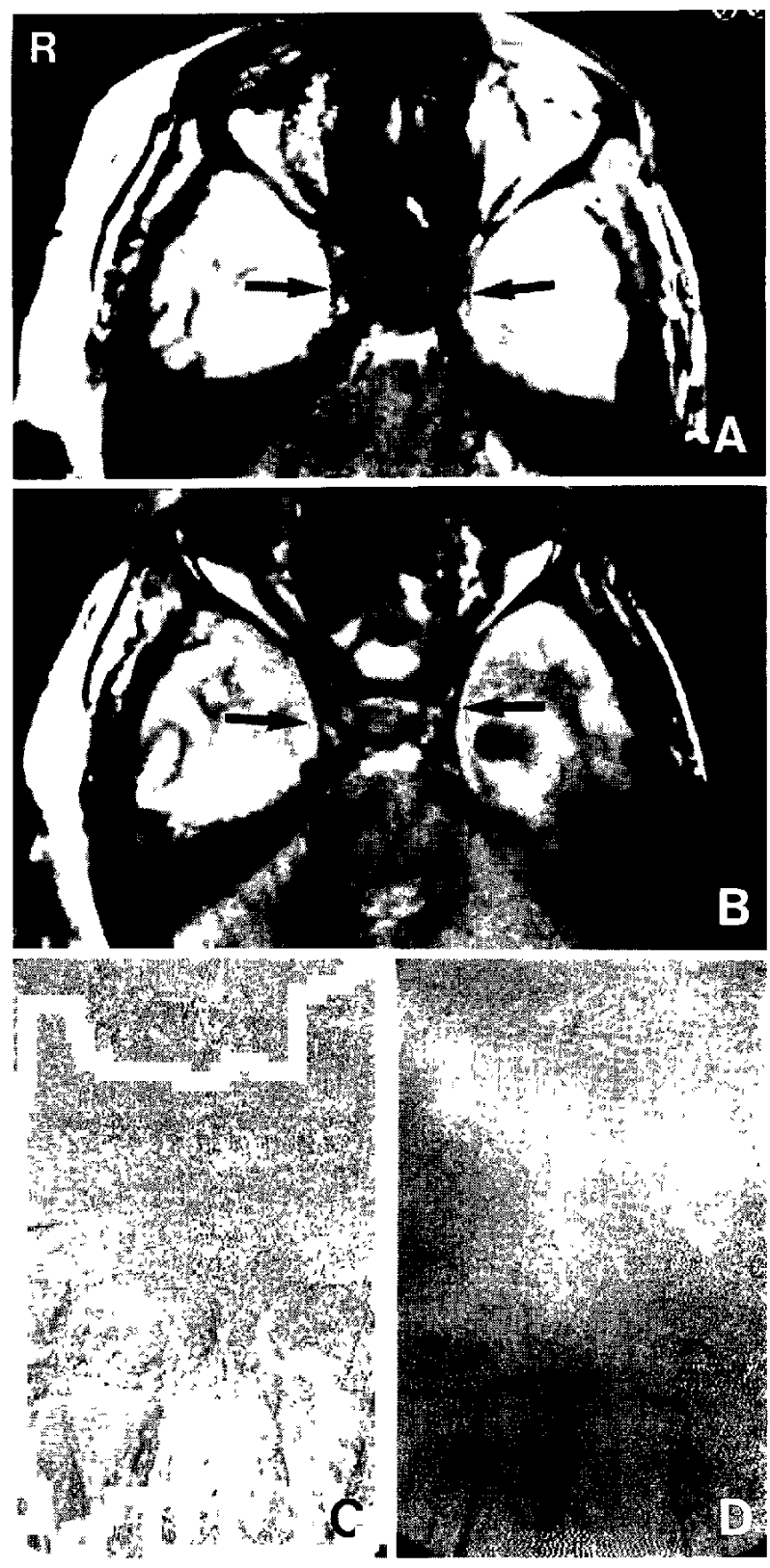

Fig. $1 T_{2}$-weighted $M R$ images showing the flow void phenomenon in the ICA (arrows) and basilar artery before brain death (A). The flow voids disappeared after brain death (B). IADSA showing the no flow phenomenon in the intracranial carotid (C) and vertebral arteries (D).

Additionally, MR imaging showed general morphological changes including massive cerebral swelling of the whole brain, and tentorial and tonsillar herniations in all patients. In patients with massive brain edema, the high intensity due to edema decreased after brain death (Fig. 3).
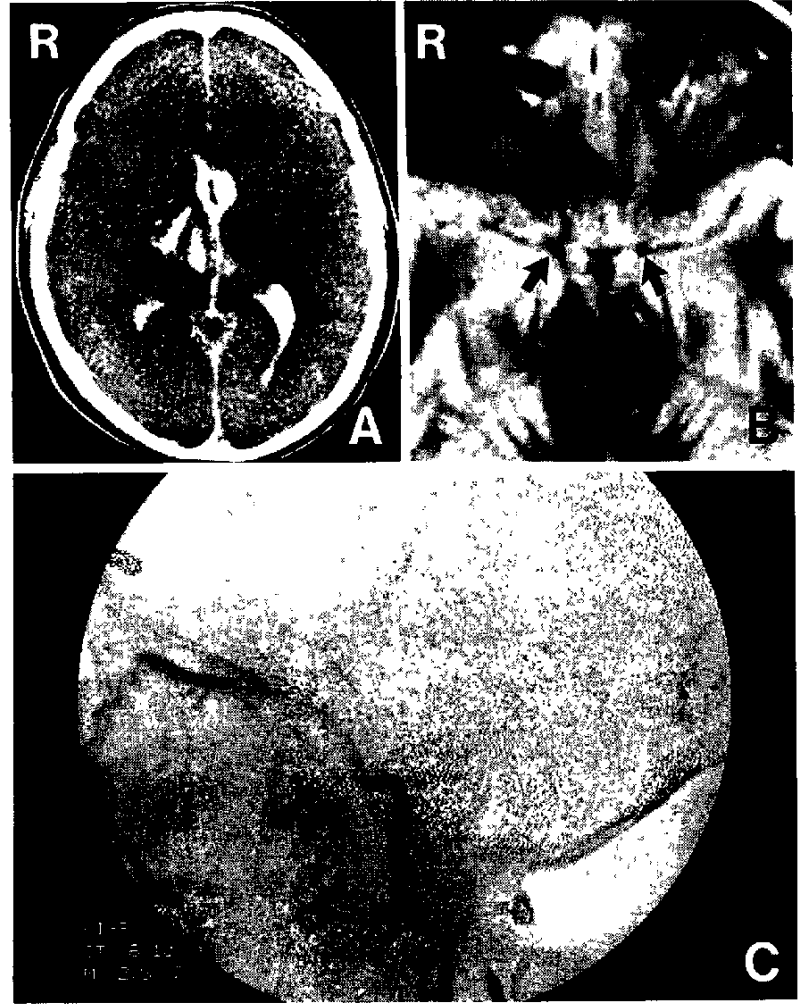

Fig. 2 A patient with brain death. CT scan showing massive thalamic and intraventricular hemorrhage (A). $T_{2}$-weighted $M R$ image indicating spotty flow voids in both carotid bifurcations (B, arrows). IADSA revealed no intracranial blood flow (C).

\section{Discussion}

The present study showed that MR imaging can demonstrate the disappearance of flow void mainly at the cavernous portion of the ICA and the major branches of the ACA or MCA. Comparison of MR imaging and IADSA revealed that non-filling of the ICA correlates with the disappearance of flow voids in the cavernous ICA. However, some spotty flow voids were seen in the MCA or ACA perpendicular to the axial slices in some patients and in the unilateral ICA in one patient, although greatly dissimilar to the normal flow void pattern. This observation of partial flow void remaining in the nonfilling brain may be explained by TCD imaging findings of a to-and-fro flow pattern in the major intracranial arteries in brain death patients. ${ }^{12,14)}$ The small but steep pulse (increased flow velocity) may cause, as an effect of time-of-flight (TOF), spotty flow voids in the vessel.

The TOF effect depends on various factors such as 


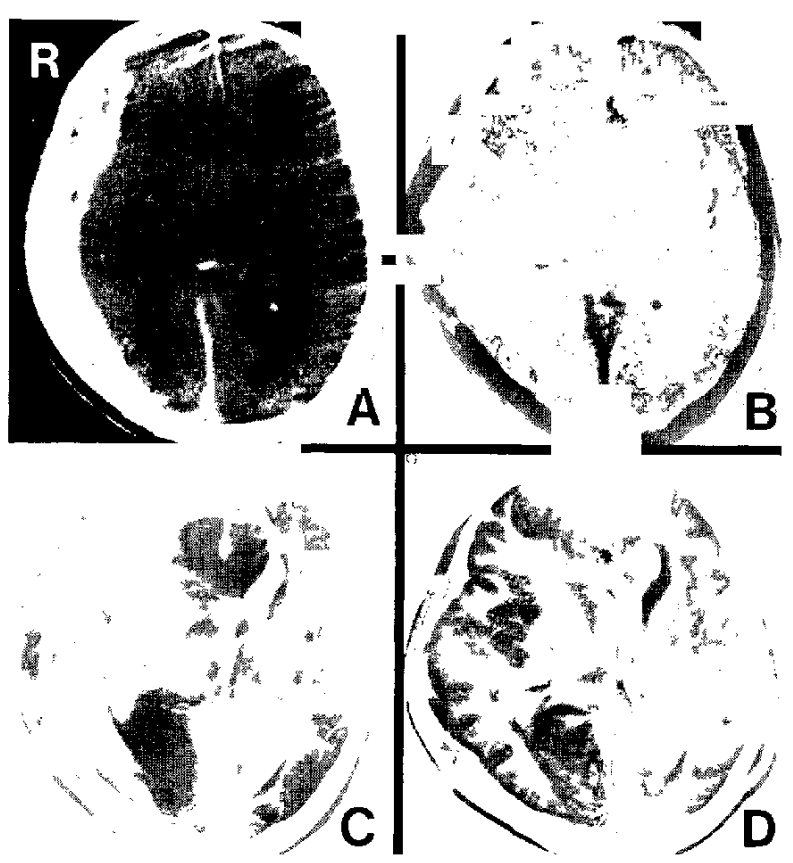

Fig. 3 CT scans before (A) and after (B) brain death showing acute subdural hematoma. $T_{2}$-weighted MR images showing the prominent edema caused by infarction due to the brain herniation before brain death $(\mathrm{C})$, which became less prominent after brain death (D). The contrast between white and gray matters increased after brain death.

the inflow effect, direction of the flow, flow velocity, pulsatility of the flow, slice thickness, and interpulse interval (half length of TE) ${ }^{4}$ so absence of flow on individual images requires careful interpretation. ${ }^{4)}$ In the SE sequence the signal vanishes if the flow velocity is greater than $2 \times$ slice thickness/TE. ${ }^{4)}$ Therefore, using a thin slice and a long TE will cover a range of slower flow velocities. However, the flow velocity in the vessels is higher than normal due to increased intracranial pressure in patients with impending brain death, ${ }^{12,14)}$ so that misinterpretation of slow flow as vascular occlusion is unlikely to occur. The TOF effect will be much less in the $M_{1}$ portion of the MCA than in the cavernous portion of the ICA since the $M_{1}$ does not run perpendicular to the axial MR slice. Horizontal blood flow will not cause the TOF effect because instead of flowing away from the slice, the blood remains within the selected slice due to the to-and-fro movement. This may also explain why the abnormal spotty flow voids were seen only in the perpendicular portion of the MCA and ACA. Serial MR imaging showed that the disappearance of the partial flow voids coincided with the disappearance of the toand-fro movement on TCD imaging in one patient (Fig. 4) which also supports this explanation. The disappearance of flow voids in the ICA was more reliable and therefore should be the basis of diagnosis of brain death using MR imaging. However, further investigations with both MR and TCD imaging in a serial study is required.

TCD imaging is useful for evaluating the intracranial flow in patients with brain death, ${ }^{12,14)}$ but accurate scans are sometimes difficult to obtain without a bone window, raising the possibility of false negative diagnosis. Patients with brain death require full monitoring and cardiorespiratory support, ${ }^{9}$ but this does not prevent investigation of such patients in the MR unit. MR angiography is a more accurate method to evaluate intracranial flow, and this has been applied to patients with brain death in a preliminary report. ${ }^{1,11)}$ Further MR angiography investigations are currently underway. ${ }^{11)}$

MR imaging can show bilateral brainstem intensity changes and brainstem distortions in severe primary brain damage patients, which are indicative of poor clinical outcome. ${ }^{(0)}$ These MR changes are more sensitive for predicting the clinical outcome than the auditory brainstem response. ${ }^{10)}$ Therefore, observation of the structural changes on MR imaging provided information about the therapeutic effects of medical therapy such as osmodiuretics, hyperventilation, or surgical decompression. Additionally, changes in the flow void pattern during the development of brain swelling were also highly informative. If normal flow void patterns are preserved despite the high intracranial pressure, a favorable outcome can be expected based on the preserved intracranial perfusion.

The relative signal intensity of white and gray matters was different in patients with brain death compared to the normal controls. The increase in contrast on $P D$ and $T_{2}$-weighted images may be explained by the different vulnerability of white and gray matters to anoxic damage or autolytic changes. Histological examinations of cases of brain death have revealed necrosis, with little or no inflammatory or cellular reaction, in contrast to other intracranial pathological situations in which blood flow is preserved. ${ }^{22}$ This unique type of the histological change is more prominent in gray than in white matter. ${ }^{22)}$ Thus, the increased contrast on PD or $T_{2^{-}}$ weighted images is thought to be due to predominantly gray matter changes. Neurons are much more vulnerable to anoxia than glia cells, and the $T_{2}$ value of neuron cytoplasm triples after cell death in vitro, ${ }^{16}{ }^{16}$ supporting the idea of pathological changes in the gray matter. However, a postmortem in vitro serial 

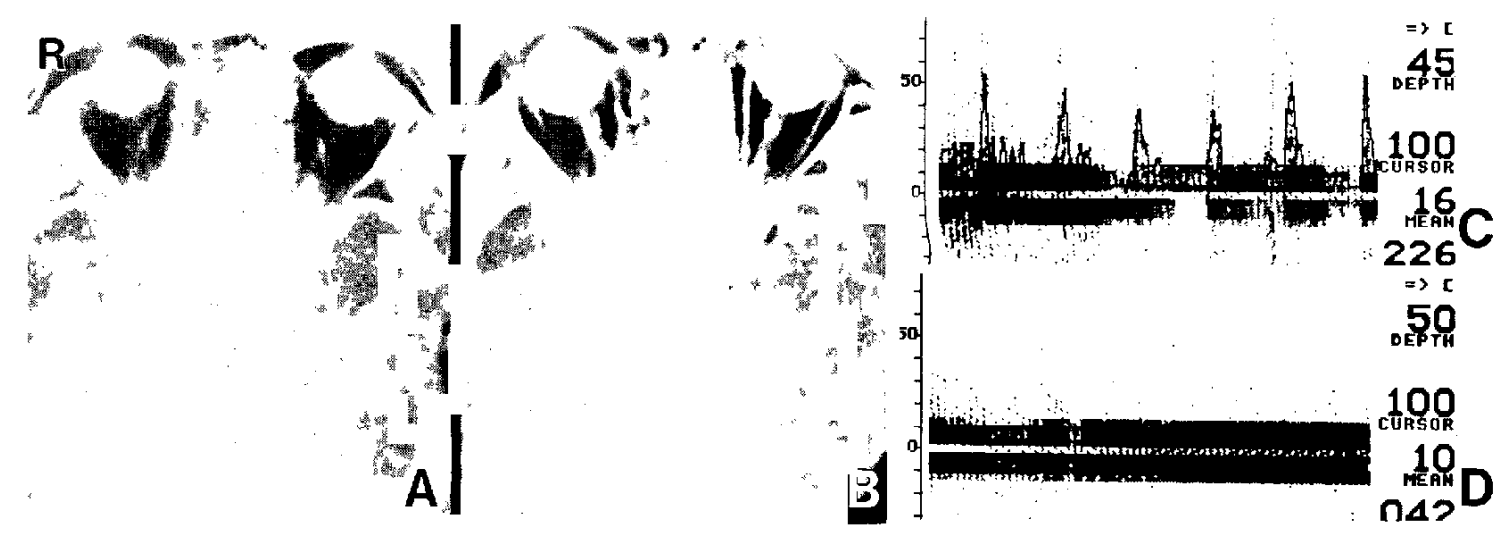

Fig. 4 PD MR images showing the presence of flow voids before brain death (A) and the absence after brain death (B). TCD scans showing a steep pulse wave in the MCA before brain death (C), and absence of flow after brain death (D).

$\mathrm{T}_{1}$ and $\mathrm{T}_{2}$ measurement of extracted porcine brain tissue maintained at under $8^{\circ} \mathrm{C}$ has revealed almost no change in $T_{1}$, but a slight decrease in $T_{2}$. The $T_{2}$ value declined at the same rate $(0.09 \mathrm{msec} / \mathrm{hr})$ in both white and gray matters. ${ }^{6}$ Other studies found no changes in $T_{1}$ and $T_{2}$ in the brain tissue, but a $T_{1}$ decrease in liver and pancreas tissue. ${ }^{3)}$ Therefore, simple postmortem autolysis is not an adequate explanation for the changes in relative intensity.

We found that perilesional edema decreased after brain death, so the water content presumably decreased in the extracellular space due to the lower perfusion pressure while cell swelling (autolysis) may have progressed as a result of energy failure predominantly in the gray matter. Since there is more extracellular space in white than in gray matter, the relative extracellular water concentration may have decreased more in white matter, thus causing the contrast increase on PD or $\mathrm{T}_{2}$-weighted images. Such changes have been also described in the acute stage of hypoxic brain damage, in which hyperintensity of the cortex and hypointensity in the white matter immediately below to the cortical area were seen. ${ }^{11}{ }^{18)}$ These selective gray matter changes may have been caused by the lower vulnerability of the cortex to hypoperfusion and energy depletion. ${ }^{18)}$ Blood flow continues in the posthypoxic brain and may cause vasogenic edema, but there is no perfusion in brain death. If these cortical changes are attributed to the same pathological mechanism, cytotoxic edema rather than vasogenic edema must be responsible. However, the changes seen are only relative and may vary in water content or behavior so that a diffusion MR study is required to distinguish vasogenic and cytotoxic edema under these conditions. ${ }^{5)}$
Our study showed that abnormal flow void patterns demonstrated by MR imaging is a potential method for the noninvasive diagnosis of brain death. The changes in the relative intensity of white and gray matters seen after brain death are attributed to the relative vulnerability to oxygen and energy depletion.

\section{Acknowledgments}

The authors express their gratitude to Mr. T. Ebashi for assistance in processing the MR data.

\section{References}

1) Aichner F, Felber S, Birbamer G, Luz G, Judmaier W, Scmutzhard E: Magnetic resonance: A noninvasive approach to metabolism, circulation, and morphology in human brain death. Ann Neurol 32: 502511,1992

2) Aliveraz O, Edwards JH, Hyman RA: MR recognition of internal carotid artery occlusion. AJNR Am J Neuroradiol 7: 359-360, 1986

3) Baba Y, Lerch MM, Stark DD, Tanimoto A, Kreft BP, Zhao L, Saluja AK, Takahashi M: Time after excision and temperature after ex vivo tissue relaxation time measurements. J Magn Reson Imaging 4: 647651, 1994

4) Bradley WG Jr: Flow phenomena, in Stark DD, Bradley WG Jr (eds): Magnetic Resonance Imaging, vol 1. St. Louis, Mosby Year Book, 1992, pp 253298

5) Cowan FM, Pennock JM, Hanrahan JD, Manji KP, Edwards AD: Early detection of cerebral infarction and hypoxic ischemic encephalopathy in neonate using diffusion weighted magnetic resonance imaging. Neuropediatrics 25: 172-175, 1994 
6) Gyöffry-Wagner Z, Englund E, Larrson EM, Brun A, Cronqvist S, Persson B: Proton magnetic resonance relaxation times $\mathrm{T} 1$ and $\mathrm{T} 2$ related to postmortem interval. An investigation on porcine brain tissue. Acta Radiol 27: 115-118, 1986

7) Handa J, Matsuda M, Matsuda I, Nakasu S: Dynamic computed tomography in brain death. Surg Neurol 17: 417-422, 1982

8) Heinz ER, Yeates AE, Djang WT: Significant extracranial carotid stenosis: Detection on routine cerebral MR images. Radiology 170: 843-848, 1989

9) Matsumura A, Meguro K, Mizutani T, Tsurushima H, Satoh N, Tsunoda T, Matsumaru $Y$, Nakata $Y$, Wada M, Kikuchi Y, Ebashi T, Nose T: Cardiorespiratory monitoring and support during MRI examination in critically ill patients. Nippon Jiki Kyomei Igakkai Zasshi 8: 268-274, 1989 (in Japanese)

10) Matsumura A, Mitsui I, Ayuzawa S, Takeuchi S, Nose T: Prediction of the reversibility of the brain stem dysfunction in head injury patients: MRI and auditory brain stem response study, in Nakamura $\mathrm{N}$, Hashimoto T, Yasue M (eds): Recent Advances in Neurotraumatology. Tokyo, Springer-Verlag, 1993, pp 192-195

11) Meguro $K$, Nakata $Y$, Narushima $K$, Wada $M$, Yokoyama Y, Matsumura A, Nose T: Assessment of the role of MRI and MRA in the diagnosis of brain death. Nippon Gazo Igaku Zasshi 11: 470, 1992 (in Japanese)

12) Powers AD, Graeber MC, Smith RR: Transcranial Doppler ultrasonography in the determination of brain death. Neurosurgery 24: 884-889, 1989

13) Rappaport ZH, Brinker RA, Rovit RL: Evaluation of brain death by contrast-enhanced computerized cranial tomography. Neurosurgery 2: 230-232, 1979

14) Ropper A, Kehne SM, Wechsler L: Transcranial Dop- pler in brain death. Neurology 37: 1733-1735, 1987

15) Rosenklint A, Jorgensen PB: Evaluation of angiographic methods in the diagnosis of brain death. Correlation with local and systemic arterial pressure and intracranial pressure. Neuroradiology 7: 215-219, 1974

16) Schoeniger JS, Aiken N, Hsu E, Blackband SJ: Relaxation-time and diffusion NMR microscopy of single neurons. J Magn Reson B 103: 261-273, 1994

17) Schwartz JA, Baxter J, Brill D, Burns JR: Radionuclide cerebral imaging confirming brain death. JAMA 249: 246-247, 1983

18) Takahashi $S$, Higano $S$, Ishii $K$, Matsumoto $K$, Sakamoto K, Iwasaki Y, Suzuki M: Hypoxic brain damage: Cortical laminar necrosis and delayed changes in white matter at sequential MR imaging. Radiology 189: 449-456, 1993

19) Tan WS, Wilbur AC, Jafar JJ, Spigos DG, Abejo R: Brain death: Use of dynamic $C T$ and intravenous digital subtraction angiography. $A J N R A m J \mathrm{Neu}$ roradiol 8: 123-125, 1987

20) Vatne K, Nakstad P, Lundar T: Digital subtraction angiography (DSA) in the evaluation of brain death. Neuroradiology 27: 155-157, 1985

21) Walker AE: An appraisal of the criteria for cerebral death. JAMA 237: 982-986, 1977

22) Walker AE, Diamond EL: The neuropathological findings in irreversible coma. A critique of the "respirator brain." J Neuropathol Exp Neurol 34: 295323,1975

Address reprint requests to: A. Matsumura, M.D., Department of Neurosurgery, Institute of Clinical Medicine, University of Tsukuba, 1-1-1 Tennodai, Tsukuba, Ibaraki 305, Japan. 